\title{
The impact of patient feedback on the medical performance of qualified doctors: a systematic review
}

Rebecca Baines ${ }^{1}$, Sam Regan de Bere ${ }^{1}$, Sebastian Stevens', Jamie Read ${ }^{1}$, Martin Marshall ${ }^{2}$, Mirza Lalani ${ }^{3}$ Marie Bryce ${ }^{1}$ and Julian Archer $^{1 *}$ (D)

\begin{abstract}
Background: Patient feedback is considered integral to quality improvement and professional development. However, while popular across the educational continuum, evidence to support its efficacy in facilitating positive behaviour change in a postgraduate setting remains unclear. This review therefore aims to explore the evidence that supports, or refutes, the impact of patient feedback on the medical performance of qualified doctors.

Methods: Electronic databases PubMed, EMBASE, Medline and PsycINFO were systematically searched for studies assessing the impact of patient feedback on medical performance published in the English language between 2006-2016. Impact was defined as a measured change in behaviour using Barr's (2000) adaptation of Kirkpatrick's four level evaluation model. Papers were quality appraised, thematically analysed and synthesised using a narrative approach.

Results: From 1,269 initial studies, 20 articles were included (qualitative ( $n=8)$; observational $(n=6)$; systematic review ( $n=3)$; mixed methodology ( $n=1)$; randomised control trial $(n=1)$; and longitudinal $(n=1)$ design). One article identified change at an organisational level (Kirkpatrick level 4); six reported a measured change in behaviour (Kirkpatrick level 3b); 12 identified self-reported change or intention to change (Kirkpatrick level 3a), and one identified knowledge or skill acquisition (Kirkpatrick level 2). No study identified a change at the highest level, an improvement in the health and wellbeing of patients. The main factors found to influence the impact of patient feedback were: specificity; perceived credibility; congruence with physician self-perceptions and performance expectations; presence of facilitation and reflection; and inclusion of narrative comments. The quality of feedback facilitation and local professional cultures also appeared integral to positive behaviour change.
\end{abstract}

Conclusion: Patient feedback can have an impact on medical performance. However, actionable change is influenced by several contextual factors and cannot simply be guaranteed. Patient feedback is likely to be more influential if it is specific, collected through credible methods and contains narrative information. Data obtained should be fed back in a way that facilitates reflective discussion and encourages the formulation of actionable behaviour change. A supportive cultural understanding of patient feedback and its intended purpose is also essential for its effective use.

Keywords: Patient feedback, Systematic review, Medical education, Impact, Behaviour change, Doctors

\footnotetext{
* Correspondence: julian.archer@plymouth.ac.uk

${ }^{1}$ Collaboration for the Advancement of Medical Education Research \&

Assessment (CAMERA), Faculty of Medicine and Dentistry, University of

Plymouth, Drake Circus, Plymouth PL4 8AA, UK

Full list of author information is available at the end of the article
}

(c) The Author(s). 2018 Open Access This article is distributed under the terms of the Creative Commons Attribution 4.0 International License (http://creativecommons.org/licenses/by/4.0/), which permits unrestricted use, distribution, and reproduction in any medium, provided you give appropriate credit to the original author(s) and the source, provide a link to the Creative Commons license, and indicate if changes were made. The Creative Commons Public Domain Dedication waiver (http://creativecommons.org/publicdomain/zero/1.0/) applies to the data made available in this article, unless otherwise stated. 


\section{Background}

Patient feedback is considered integral to quality improvement and professional development [1-3]. Designed to guide behaviour change and facilitate reflective practice [4], patient feedback is increasingly incorporated into medical education including continuing professional development and regulatory initiatives such as medical revalidation [5-9]. Typically collected as part of a questionnaire based assessment $[10,11]$, patient feedback tools have been validated across a range of specialities and geographical locations including Canada, the USA, Netherlands and Denmark [10]. However, their inclusion in regulatory initiatives and other educational activities is not without its criticisms, with current literature to support its impact on medical performance particularly limited in a post-graduate setting $[11,12]$.

Kluger and others critique the importance placed on patient feedback as a performance assessment methodology due to the implicit and often unclear assumptions made about its capacity to facilitate quality improvement [11, 13-15]. The quality of evidence used to support its capacity to facilitate change is also frequently called into question [13]. As Lockyer et al. notes, notwithstanding the considerable amount of research examining the psychometric properties of specific patient feedback tools, current understanding of patient feedback as a catalyst for change remains limited [12]. Little attention has been paid to the formative or educational impact of patient feedback on doctor performance [10, 11, 16, 17]. As Ferguson and others note, further research is needed to establish if, and how, patient feedback influences doctor i.e. physician or resident behaviour and to identify which factors may have greatest influence [13].

As a result, in line with international efforts to incorporate patient feedback into regulatory and other educational initiatives [7, 18], we undertook a systematic review to: i) assess if, and how, patient feedback is used by the medical profession; ii) identify factors influential in determining its efficacy and; iii) identify any potential challenges or facilitators surrounding its impact on medical performance. Our review specifically sought to address the following research questions: what impact does patient feedback have on the medical performance of individual doctors, and what factors influence its acceptance in a medical environment?

For this review, we use the term 'patient' to be inclusive of service-users, consumers, carers and/or family members although the important distinctions between these terms is acknowledged. We define patient feedback as information provided about an individual doctor through formal patient experience or satisfaction surveys/questionnaires e.g. multi-source feedback (MSF) or patient feedback assessments but exclusive of formal complaints, online platforms or feedback beyond the service of an individual doctor i.e. healthcare team or service.

\section{Methods}

To ensure transparency of findings, our review followed the Preferred Reporting Items for Systematic Reviews and Meta-Analysis (PRISMA) [19], and Centre for Reviews and Dissemination guidance [20].

\section{Search Strategy}

Using the SPICE framework [21], one research team member (RB) designed the search terms listed in Table 1. All search terms were reviewed by the wider team in line with the Peer Review of Electronic Search Strategies (PRESS) guidance to maximise sensitivity and specificity [22]. As advised by an information specialist, we searched Medline, EMBASE, PsycINFO and PubMed databases for articles published in the English Language between January 2006 and December 2016. This date parameter was selected to ensure the most contemporary information was included. Electronic searches were supplemented with citation searches and reviewing reference lists of eligible studies. Duplicate studies were removed electronically and double checked by another research team member (SS). Two independent reviewers conducted the research process.

\section{Study selection}

We selected studies through a two-stage process. Firstly, two reviewers (RB, SS) independently examined titles and abstracts using Rayyan, a web application for systematic reviews [23]. To ensure inclusion/exclusion standardisation, reviewers used a piloted inclusion criteria form [Additional file 1]. When a selection decision could not be made, the full article was retrieved. Potentially relevant articles were then independently assessed by two researchers (RB, SS). If any discrepancies arose these would have been resolved by discussion with a

Table 1 Systematic review search strategy

Search strategy

Setting: "physician" OR "doctor" OR "surgeon"

AND

Perspective: "doctor" OR "physician" OR "surgeon" OR "patient*" OR

"user" OR "client" OR "consumer*" OR "survivor" OR "representative*" OR "family" OR "relative"

AND

Intervention: "multisource feedback" OR "multi-source feedback" OR "360 degree feedback" OR "360 degree evaluation" OR "MSF" OR "performance feedback" OR "PF" OR "patient experience" OR "patient survey" OR "patient questionnaire"

AND

Evaluation: "professional development" OR "behaviour change" OR "improve" OR "quality of care" OR "learn*" OR "reflect" OR "impact" OR "outcome" OR "patient safety" 
third reviewer (JR) until consensus was achieved. This process was not required during the research process.

\section{Inclusion/exclusion criteria}

Studies published in the English language between 2006-2016, exploring the impact of patient feedback on medical performance in any healthcare setting using any study design except opinion, commentary or letter articles were included. Due to resource constraints studies published in languages other than English were excluded, as were those outside the pre-defined date parameters to ensure only the most contemporary evidence was reviewed. Studies that solely discussed the psychometric properties of specific patient feedback tools were excluded due to the review focusing on reported change in medical performance.

Where studies discussed the impact of MSF or work-placed based assessment more broadly but included findings about patient feedback which could be clearly identified, these were included. If it was not possible to differentiate the specific influence of patient feedback from other feedback sources, the article was excluded to avoid result dilution. Finally, due to our area of interest, studies in the context of undergraduate medical education and methods of patient feedback not currently accepted in regulatory processes such as online feedback sites were excluded.

\section{Data extraction and outcomes}

Two reviewers (RB, SS) independently undertook data extraction of all included studies using a piloted data extraction form. Information extracted included: year published; study location, aim design, population, and methodology. In order to address our research questions, we used Barr's (2000) adaptation of Kirkpatrick's four level evaluation model [Additional file 2] to evaluate study outcomes [24]. Where studies covered MSF or work-place based assessments more broadly, only those findings relating specifically to patient feedback were extracted for review inclusion.

\section{Quality assessment}

Two research team members (RB, SS) independently assessed study quality using: the Critical Appraisal Skills Programme Qualitative checklist [25]; Quality Assessment instrument for observational cohort and cross-sectional studies [26]; and Quality Assessment of Systematic reviews and Meta-analyses [27]. Due to the focus of this review not relying solely on the methodological quality of included studies, conceptual relevance took precedence over methodological rigour [28]. However, we conducted sensitivity analyses to assess the impact of study quality on review findings [29, 30]. Sensitivity analyses test for the effect of study inclusion/ exclusion on review findings [29]. It is considered an important focus of any review synthesis involving qualitative research [31].

\section{Data analysis and synthesis}

Data were analysed using an inductive thematic analysis approach [29, 32]. The team initially reviewed two papers to develop a comprehensive coding framework. The framework was then used to individually analyse all included studies and to iteratively compare emerging themes across studies to determine dominant themes. We then synthesised themes using a modified narrative synthesis technique grounded in Popay et al's. guidance [33].

\section{Results}

From an initial identification of 1,269 articles, 36 studies were considered potentially relevant. Of these, 18 were excluded due to irrelevant: study design [34]; intervention; [6, 35-42] or outcome i.e. did not discuss medical performance impact. [5, 16, 43-49] A total of 18 articles supplemented with two articles found through reference list searching were included for the purposes of this review (Fig. 1). Results are discussed in order of study characteristics; study quality; impacts of patient feedback on medical performance; and factors found to influence the use of patient feedback to improve medical performance.

\section{Study characteristics}

We included studies with a variety of study designs including: qualitative methodologies $(n=8)$; observational designs $(n=6)$; systematic reviews $(n=3)$; mixed methodologies $(n=1)$; one randomised control trial; and a longitudinal study. Studies primarily focused on family doctors $(n=6)$. Other populations studied included: unspecified doctor groups or settings $(n=5)$; residents $(n=3)$; consultants $(n=2)$; medical specialists $(n=1)$; primary care groups leaders $(n=1)$, doctors $(n=1)$; and department chiefs $(n=1)$. Studies were conducted in: Canada $(n=6)$; the UK $(n=5)$; US $(n=4)$; Netherlands $(n=4)$ and Denmark $(n=1)$. A summary of study characteristics is provided in Table 2.

\section{Study quality}

We found the methodological quality of included studies to be mixed. Studies were considered high $(n=13)$, acceptable $(n=6)$, and low $(n=1)$. Although low, sensitivity analysis identified that its inclusion had no impact on the review synthesis and did not therefore dilute conclusions drawn. 


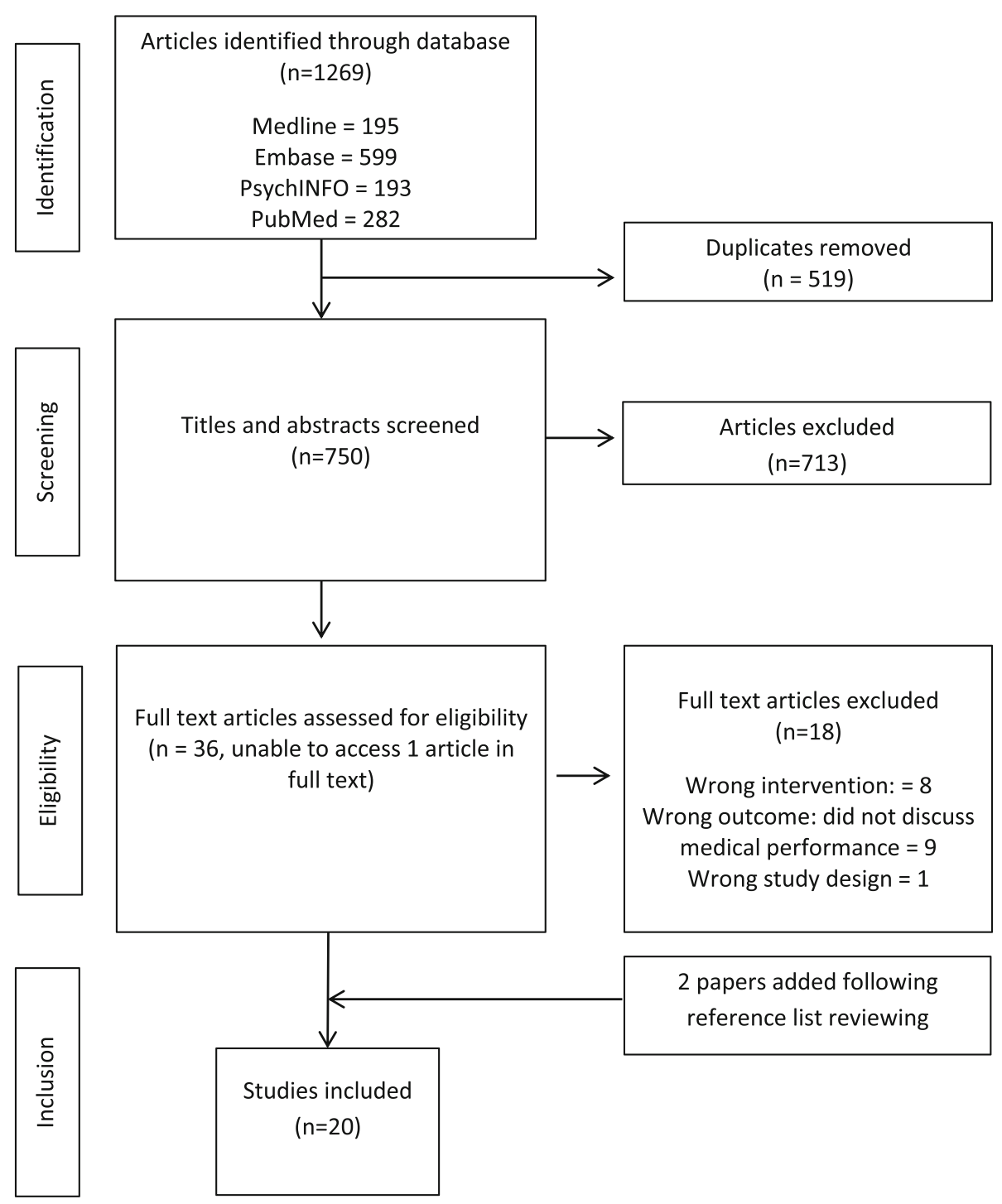

Fig. 1 PRISMA flow diagram

Impact of patient feedback on medical performance Included studies reported: a change in organisational practice ( $n=1$, Kirkpatrick level 4a) [7]; a measured change in behaviour ( $n=6$, Kirkpatrick level 3b) [13, 50-54]; self-reported change or intention to change ( $n=12$, Kirkpatrick level 3a), [2, 8, 10, 11, 17, 55-61] and acquisition of knowledge or skills $(n=1$, Kirkpatrick level 2b) following the provision of patient feedback [1]. No studies identified a change at the highest evaluation level - a change in the health and wellbeing of patients (Kirkpatrick level 4b). These are discussed in turn below.

\section{Measured change}

We found mixed results for the evidence of measured change in medical performance. For example, an RCT demonstrated an increase in patient ratings for both the control $(n=18)$, and intervention group $(n=18)$ on items relating to communication and shared decisions [50]. Participants in the intervention group (who participated in tailored coaching sessions) also experienced statistically significant increases in four additional items: being friendly; being respectful; showing interest; and explaining problems. However, in contrast to nurse ratings, the overall difference in patient ratings between groups did not reach statistical significance [50]. It is unclear which element of the MSF intervention e.g. the MSF itself or tailored coaching led to the measured changes, preventing an assessment of potential causation. A longitudinal study investigating changes in medical performance as assessed by patients, co-workers and medical colleagues also identified significant changes (small-moderate) in 


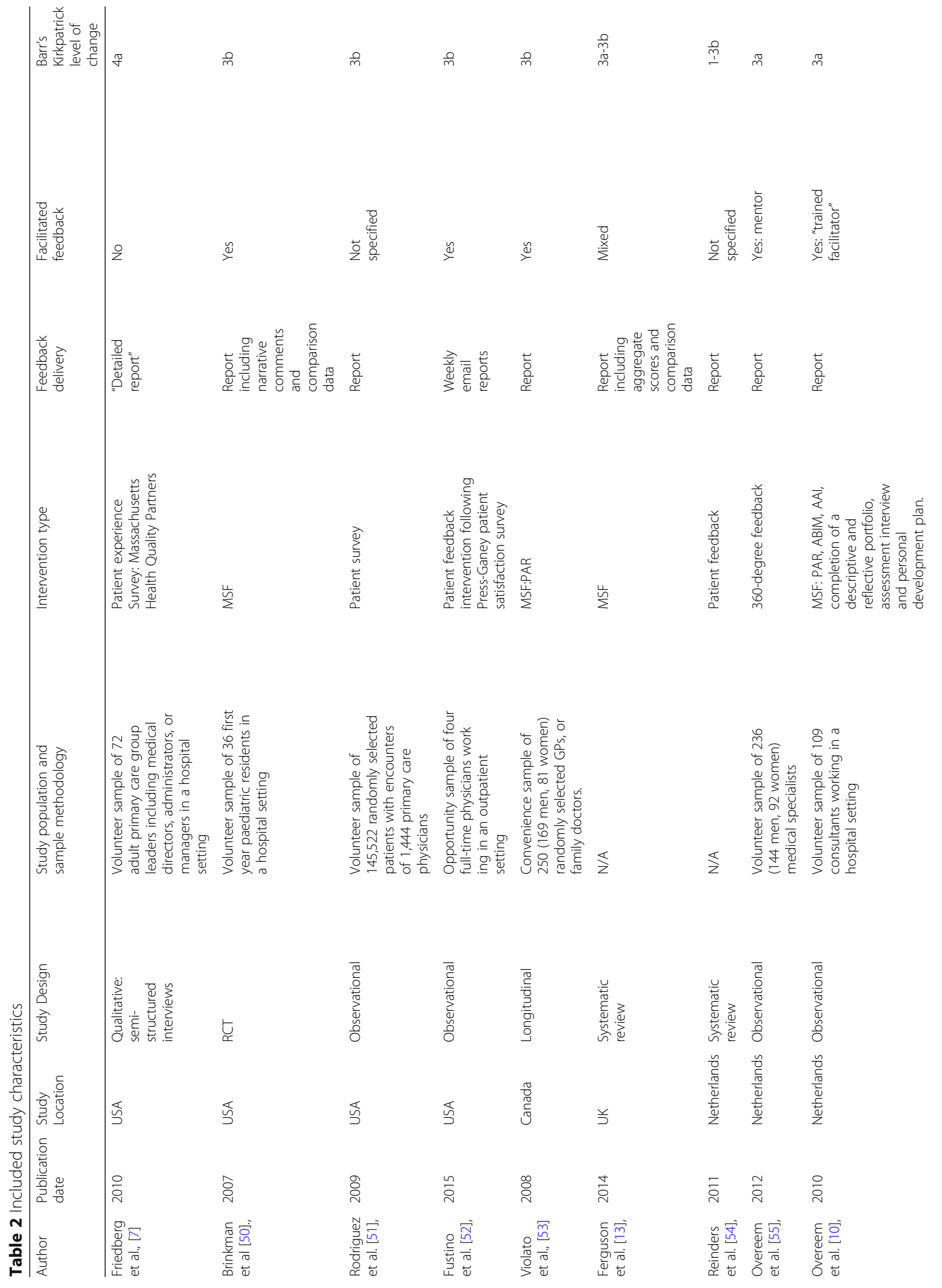




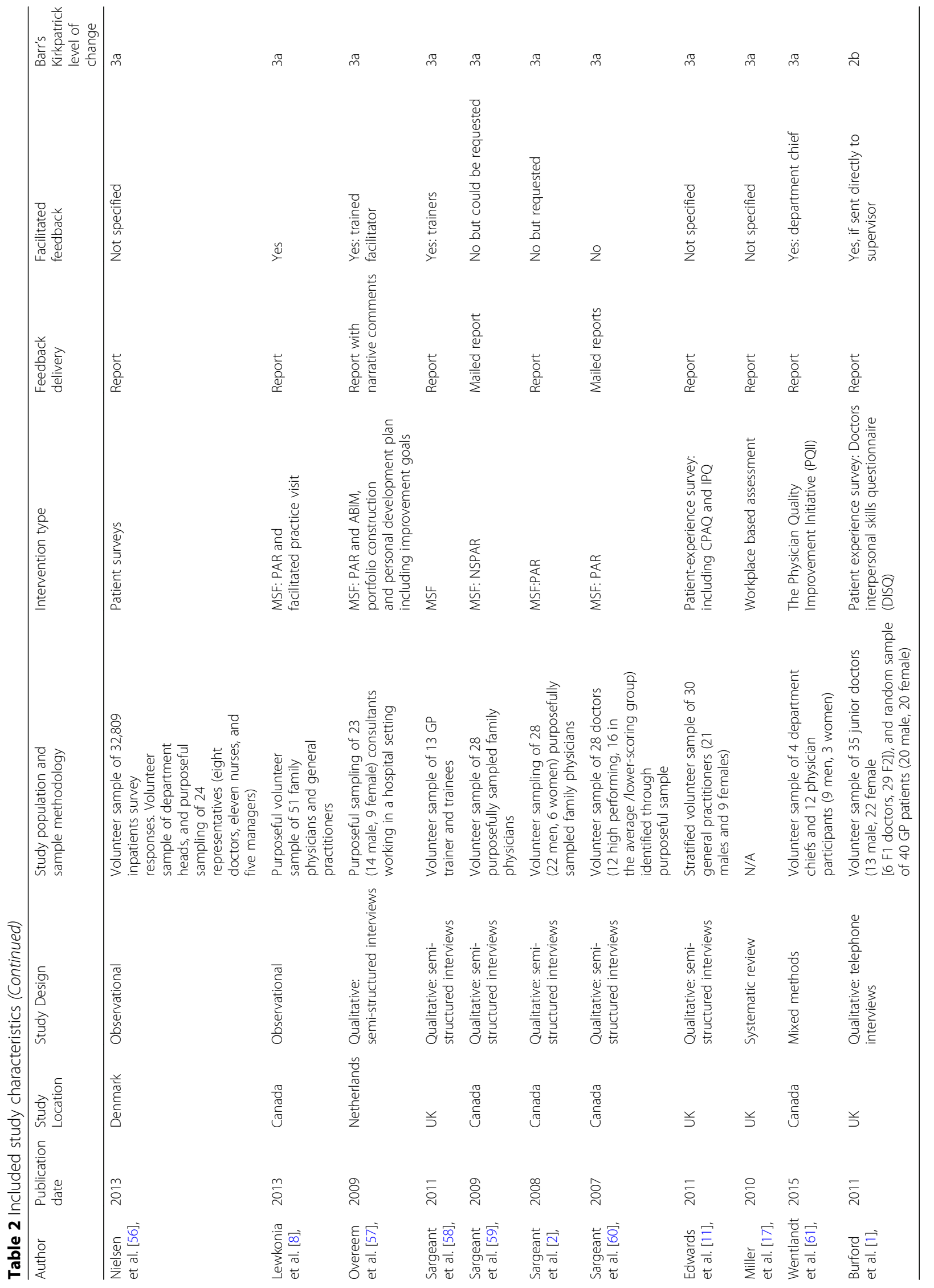


colleague and co-worker assessments, but not that of patient feedback [53].

Conversely, other studies reported significant improvements in patient feedback [51, 52, 54]. One observational study assessing the impact of financial incentives on patient experience for individual doctors identified significant improvements in: doctor-patient communication [95\% confidence interval (CI): $0.61,0.87, p<0.001$ ]; care coordination $(0.48 ; 95 \%$ CI: $0.26,0.69)$; and office staff interaction (annual point change $=0.22 ; 95 \% \mathrm{CI}$ : $0.04,0.40, p=0.02$ ) over a period of three years [51]. Doctors with lower baseline performance scores typically experienced greater improvements $(p<0.001)$. Similarly, incentives that placed greater emphasis on clinical quality and patient experience were associated with larger improvements in care coordination $(p<0.01)$ and office staff interaction $(p<0.01)$. In contrast, incentives emphasising productivity and efficiency were associated with declines in doctor communication performance $(p<0.01)$ and office staff interaction $(p<0.01)[51]$.

\section{Self-reported change}

Similar to the results of measured change studies, self-report studies appear mixed in terms of patient feedback use and efficiency and typically identify a small-moderate change. $[2,8,55,58,60]$ In one study, $78 \%(40 / 51)$ of primary care doctors reported making a practice change following patient feedback results [8], but most included studies reported a smaller effect $[55,60]$. For example, in one study where participants received average-lower scores (13/28), $54 \%(7 / 13)$ reported making a change [60]. However, $54 \%(15 / 28)$ of participants from the same study also reported making no change; [60] highlighting the variability of patient feedback impact $[2,13,17,57$, $59,60]$. Some included studies reported no intention to change $[10,57,61]$.

\section{Change in knowledge/skill acquisition}

One study identified a change in knowledge acquisition/ understanding [1]. Doctors involved in this study reported learning about the importance of trust, consultation style and communication [1].

\section{Improvements or changes made}

Finally, while all 20 studies reported a change in medical performance to some degree, 13 identified specific changes in behaviour. Communication was the most frequently targeted area for improvement $[1,7,13,50-54$, $60,61]$. Few identified initiatives targeting clinical competence, care coordination [51], or access to healthcare services $[7,52]$.

\section{Factors found to influence the use of patient feedback to improve medical performance}

Several studies identify the source, content and delivery of patient feedback to be influential in its assimilation, acceptance and use. Specifically, its: perceived credibility; congruency with self-perceptions and performance expectations; presence of facilitation and reflective discussions; and inclusion of narrative comments.

\section{Feedback source}

Nine studies reviewed described the perceived credibility of patient feedback as influential $[2,8,10,11,13,17,56$, $58,60]$, particularly when feedback was considered negative in nature $[2,11,13]$. Doctors who received negative feedback typically placed greater emphasis on the assessment process; often citing such factors as reasons behind non-acceptance [2]. Similar findings are also reported in Ferguson et al.'s review where doctors questioned feedback credibility and postponed behavioural change until the process had been verified by conducting their own independent reviews [13].

Feedback is also more likely to be incorporated into behaviour change efforts when a doctor considers the rater to be familiar and able to observe their practice $[13,17,60]$. Sargeant et al. reported that doctors who made a change did so in response to patient feedback preferentially over that of medical colleagues [60]. Conversely, research conducted by Edwards et al., identified ambiguity surrounding the credibility of patient feedback [11]. Doctors interviewed highlighted concerns that patients were completing feedback surveys on the basis of their general views of the doctor as a person and not that of their medical performance [11]. Similarly, Overeem reported that only the mean ratings of colleagues $(\mathrm{r}=-0.195, p<0.01)$ and self-ratings $(\mathrm{r}=-0.179, p<0.05)$ and not those of patients were significantly correlated with reported behaviour change [10].

\section{Feedback content}

Factors identified as influential in terms of feedback content included: feedback specificity; a perceived need for change; and consistency with other feedback sources $[2,11,13,53,60,61]$. However, we found that the most influential factor identified by eight included studies was feedback congruency between a doctors' self-perception and performance expectation $[2,10,11,13,55,58-60]$. As described by Sargeant et al., feedback interpreted as positive is typically congruent with ones' self-perception or expectations whereas, feedback interpreted as negative is typically incongruent with such perceptions [59]. Both forms of feedback may be troublesome to incorporate into behaviour change [11, 58-60]. Edwards et al., reported that feedback considered above average, i.e. positive, rarely led to actionable change as it was simply 
considered a positive affirmation of practice [11]. Conversely, negative feedback, tends to elicit greater emotional reactions and extended periods of reflection, that may, or may not, led to eventual acceptance [59]. For example, doctors interviewed two years after receiving feedback inconsistent with self-perceptions reported the same emotional and reflective reactions as experienced two years before [2].

\section{Feedback delivery: facilitation and reflection}

Early access to facilitated reflective discussions that explore emotional reactions appear integral to feedback assimilation, acceptance and subsequent use $[2,10,11,13$, $17,58,59,61,62]$. Several studies described how facilitation can support feedback acceptance and encourage achievable goal setting $[2,10,13]$. Studies that failed to provide facilitated feedback indicated a need for such an activity [13]. In one instance, a series of recorded discussions between trainees and trainers about a MSF report found trainers used open-ended questions to initiate reflective discussions and subsequent behaviour change initiatives [58]. Such openness and encouragement was widely appreciated by interviewed trainees and accepted as a way to enable unanticipated learning [58]. Identified benefits specifically related to facilitated reflective discussions include: reduced anxiety; more timely processing of patient feedback; validation of emotional reactions; prevention of jumping to premature or potentially incorrect conclusions; and increased ability to identify specific change needs $[58,59,61]$.

\section{Facilitation quality}

However, perceived mentor quality can limit the facilitation of patient feedback [58, 61]. Research conducted by Overeem et al., suggests consultants who identified specific facilitator skills including reflection, encouragement and specificity in goal setting were key to behavioural change [10]. Consultants who attained higher levels of improvement regularly identified these facilitator skills [10].

\section{Narrative comments}

The inclusion of narrative comments was influential in supporting behaviour change $[10,13,58]$. Evidence reviewed suggests participants prefer to receive written comments as opposed to numerical scores only, and that there is a small, yet significant, preference for free text comments, with written comments from raters considered essential to physician satisfaction and patient feedback use [13]. Furthermore, an analysis of interview transcripts discussing MSF reports by Sargeant et al., reveals that trainers and trainees do not typically discuss the numerical scores, but focus their discussion predominately on the narrative comments provided [58].

\section{Medical culture}

The existing medical culture may complicate behaviour change efforts $[2,10,56,57]$. As acknowledged by Nielsen et al., norms that originate within the medical community, including a lack of openness and social support, may restrict performance initiatives [56]. Sargeant et al. described how many doctors interviewed discussed the influential nature of the professional culture on performance expectation and subsequent feedback acceptance [60]. Participants spoke of "being a doctor" and how this identity made it particularly important to be viewed positively by others. The authors explain how the collective, and individual desire for doctors to "do good," leads to doctors holding a high expectation of providing above average care [60]. Feedback that challenges this self-perception is then often difficult to assimilate. Furthermore, while self-directed practice is considered the norm in medicine, being assessed in practice is typically not [2].

Finally, Nielsen argues that hospital environments and other medical settings leave little room for rational patient-centred change, due to competition with other more clearly specified institutional norms [56]. Overeem reports that consultants are not strongly motivated to use feedback to improve medical performance as they see feedback exercises as a means to enhance public trust, and not one to incentivise performance improvement $[10,57]$. Overeem concludes that one of the most frequently experienced barriers to behavioural change is working in an environment unconducive to lifelong reflective learning $[10,57]$.

\section{Discussion}

Our review responds to calls for further research to establish if, and how, patient feedback impacts on medical performance and to identify factors influential in this process [63]. While several existing reviews have explored the impact of workplace based assessments and MSF more broadly, to date, no reviews have focussed specifically on the educational impact of patient feedback beyond consultation or communication skills. Our review findings suggest patient feedback has the potential to improve medical performance, but the level at which behaviour change occurs as assessed by Kirkpatrick's evaluation model varies. No included study identified a change at the highest evaluation level, a change in the health and wellbeing of patients. Longer term studies that explore the relationship between patient feedback and impact are needed, as is the examination on patient wellbeing, although the difficulties of achieving this are acknowledged [17, 54].

Our proposed explanation for the behavioural change variability reported is the presence, or absence, of factors identified as influential in patient feedback acceptance, 
use and assimilation. Specifically, its: perceived credibility; specificity; congruency; presence of facilitated and reflective discussion; and inclusion of narrative comments. Patient feedback is more likely to initiate behaviour change if participants: consider the process, instrument and provider to be credible; receive feedback that is consistent with self-perceptions or performance expectations; are able to identify specific behavioural change measures through reflective discussions; discuss their feedback with a skilled facilitator who use open ended questions to facilitate reflective discussions and behaviour change and receive narrative comments.

The value of narrative feedback is acknowledged across postgraduate and undergraduate settings due to the unadulterated information they provide over and above that provided in numerical scores or grades [64-66]. Although not without its difficulties, $[67,68]$ there is increasing evidence to suggest recipients can interpret comments and use them to modify their performance $[69,70]$. Recent research also highlights the "stark contrast between survey scores and comments provided" [64], with patients often awarding highly positive or inflated scores [66], in addition to conflicting negative narrative comments. A focus on inflated scores could mislead professional development efforts and diminish the apparent need for continued improvement. Opportunities for reflective learning and professional development may therefore lie in narrative feedback as opposed to numerical scores, an element existing feedback tools currently rely on with limited scope or room for narrative feedback inclusion. Similar to Sargeant et al.'s research in a trainee setting, future research should examine the content and focus of feedback discussions when reviewing patient feedback reports. Is there an equal discussion between the numerical scores and narrative comments, or does one domain take precedence over the other? Based on the evidence reviewed, narrative feedback should be incorporated into current and future feedback tools across the education continuum to encourage reflective practice and beneficial behaviour change where required.

As part of the contextual landscape in which patient feedback is received, we found that facilitated reflection appears integral to transforming initial patient feedback reactions into measurable behavioural change, quality improvement initiatives or educational tasks [11, 58]. With this in mind, receiving feedback in isolation of reflective and facilitated discussions may not be enough to bring about immediate or sustained change to the betterment of professional development and subsequent patient care [71]. This alongside the highlighted importance of facilitator quality has important implications for the recent Pearson review into medical revalidation in the UK where the importance of reflective discussions was identified; "it's [feedback] only useful if the quality of the appraiser/appraisal is good and there is appropriate reflection at appraisal." [18] Facilitated discussions where reflection is supportively encouraged appears integral to dealing with emotional responses and transforming initial reactions into measurable behavioural change.

Finally, one factor that appears relatively unexplored in the existing literature is the influence of cultural context [71]. Encouraging a culture that promotes constructive feedback and reflection-in-action could enable performance improvement more readily [10, 58]. As reported by Pearson, medical revalidation is currently "at the acceptance stage, and the next step is to strengthen ownership by the profession, and engagement with the

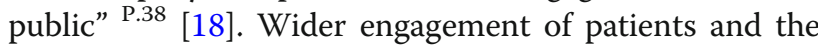
public as suggested may provide the cultural change catalyst needed to support behavioural change and educational outcomes. However, it is notable that we did not find any literature on patient feedback from a patient perspective. Assumptions are often made about the desire of patients and the public to feedback on their doctors i.e. on what, how and when, but exploration of these issues have been little explored. Organisations and institutions that use patient feedback as a form of performance evaluation should seek to alter existing cultures, enabling the collection of patient feedback to become a valued and embedded activity. This will need to include an honest and protected space in which to allow doctors to openly reflect and where needed, acknowledge error without fear and consequence [72, 73].

Strengths of this review include its application of a recognised systematic review process, $[19,20]$ and utilisation of Kirkpatrick's evaluation model to provide greater insight into the impact of patient feedback. However, its limitations must also be acknowledged. The methodological quality of some included studies is somewhat undermined by the voluntary nature, and in some cases, small sample size of participant populations. Acknowledged limitations of this sampling method include potentially biased or highly motivated participants whose results may not generalise to the wider population. Most studies are also non-comparative or observational. The conclusions drawn may therefore be limited by their uncontrolled nature. However, assessing behavioural or educational impact on the medical performance of individual doctors is difficult to achieve [54]. For example, few studies differentiate between medical practice and educational improvements, or clearly define these parameters. Furthermore, descriptive or observational studies provide useful information in the exploration of complex interactions therefore warranting their inclusion [17]. The predominance of qualitative or observational methodologies in this review should not therefore be seen as a significant limitation. Despite its frequent use in medical education, Kirkpatrick's framework 
is also not without its critics [74, 75]. Furthermore, three studies by Sargeant et al. [2, 59, 60], draw on the same sample population leading to possible publication bias. Some systematic reviews included in this article also report on the same primary studies, leading to possible result duplication. Finally, although an extensive review of published literature was undertaken, grey literature was not included and relevant non-peer reviewed studies may therefore not be included.

Future research should explore the feasibility of conducting a realist review [76] to help further unpick the complexity of patient feedback and to identify what works for whom, and in what circumstances. Realist reviews are increasingly being adopted in other areas of medical education including doctor appraisal [77] and internet based education [78].. To the authors' knowledge, a realist review of patient feedback in medical education has yet to be completed highlighting a gap in existing knowledge.

\section{Conclusion}

This review holds import implications for the use of patient feedback across the educational continuum. Patient feedback can have an impact on medical performance. However, its acceptance, assimilation, and resultant change, are influenced by a multitude of contextual factors. To strengthen patient feedback as an educational tool, initiatives should be: specific; collected through credible methods; contain narrative comments; and involve facilitated reflective discussions where initial emotional reactions are processed into specific behavioural change, quality improvement initiatives or educational tasks. Understanding and encouraging cultural contexts that support patient feedback as an integral component of quality improvement and professional development is essential. Future patient feedback assessment tools should be accompanied by facilitated discussion that is of high quality.

\section{Additional files}

Additional file 1. Piloted inclusion form. (DOCX $16 \mathrm{~kb}$ )

Additional file 2. Barr's (2000) adaptation of Kirkpatrick's four level evaluation model. (DOCX $15 \mathrm{~kb}$ )

\section{Acknowledgements}

The authors would like to thank the information specialist at Plymouth University, all UMbRELLA collaborators (www.umbrella-revalidation.org.uk) and the national PPI forum.

\section{Funding}

This research was funded by the General Medical Council (GMC 152). The funding body had no involvement in the study design, data collection, analysis or interpretation. The funding body was not involved in the writing of this manuscript. The views expressed in this report are those of the authors and do not necessarily reflect those of the General Medical Council.

\section{Availability of data and materials}

Data used in this study are freely available as all papers can be found in the published literature and are listed in the papers reference list. The datasets used and/or analysed are available from the corresponding author on reasonable request.

\section{Authors' contributions}

JA and SRdB conceived the study as part of a wider research programme into medical revalidation (UMbRELLA www.umbrella-revalidation.org.uk). RB formulated the review's design with critical input from all the other authors. $R B, S S$ and JR undertook the review and critically appraised the literature. RB wrote the first draft of the paper with multiple iterations commented on and shaped by other authors. All authors read and approved the final manuscript.

Ethics approval and consent to participate

This literature review was exempt from requiring ethical approval.

\section{Consent for publication}

Not applicable

\section{Competing interests}

Professor Julian Archer (4438050), Dr Jamie Read (7083368) and Professor Martin Marshall (3243842) are registered and hold a licence to practise with the GMC. Dr Jamie Read is also a GMC Associate. GMC Associates are an appointed group made up of medics (both qualified and trainees) and nonmedical (lay) experts to partake in a number of activities across the organisation.

\section{Publisher's Note}

Springer Nature remains neutral with regard to jurisdictional claims in published maps and institutional affiliations.

\section{Author details}

${ }^{1}$ Collaboration for the Advancement of Medical Education Research \& Assessment (CAMERA), Faculty of Medicine and Dentistry, University of Plymouth, Drake Circus, Plymouth PL4 8AA, UK. ${ }^{2}$ Improvement Science London, University College London, London, UK. ${ }^{3}$ Research Department of Primary Care and Population Health, University College London, London, UK.

Received: 27 June 2017 Accepted: 11 July 2018

Published online: 31 July 2018

References

1. Burford B, Greco M, Bedi A, Kergon C, Morrow G, Livingston M, Illing J. Does questionnaire-based patient feedback reflect the important qualities of clinical consultations? Context, benefits and risks. Patient Educ. Couns. 2011; 84(2):e28-36.

2. Sargeant J, Mann K, Sinclair D, Van der Vleuten C, Metsemakers J. Understanding the influence of emotions and reflection upon multi-source feedback acceptance and use. Adv Health Sci Educ Theory Pract. 2008;13(3): 275-88.

3. Griffiths A, Leaver MP. Wisdom of patients: predicting the quality of care using aggregated patient feedback. BMJ Qual Saf. 2018;27(2):110-8.

4. Sargeant J, Macleod T, Sinclair D, Power M. How do physicians assess their family physician colleagues' performance?: creating a rubric to inform assessment and feedback. J Contin Educ Health Prof. 2011;31(2):87-94.

5. Lee VS, Miller T, Daniels C, Paine M, Gresh B, Betz AL. Creating the Exceptional Patient Experience in One Academic Health System. Acad Med. 2016;91(3):338-44

6. Nurudeen SM, Kwakye G, Berry WR, Chaikof EL, Lillemoe KD, Millham F, Rubin M, Schwaitzberg S, Shamberger RC, Zinner MJ, et al. Can 360-Degree Reviews Help Surgeons? Evaluation of Multisource Feedback for Surgeons in a Multi-Institutional Quality Improvement Project. J Am Coll Surg. 2015; 221(4):837-44

7. Friedberg MW, SteelFisher GK, Karp M, Schneider EC. Physician groups' use of data from patient experience surveys. J Gen Intern Med. 2011;26(5): 498-504

8. Lewkonia R, Flook N, Donoff M, Lockyer J. Family physician practice visits arising from the Alberta Physician Achievement Review. BMC Med Educ. 2013;13:121 
9. Hill JJ, Asprey A, Richards SH, Campbell JL. Multisource feedback questionnaires in appraisal and for revalidation: a qualitative study in UK general practice. Br J Gen Pract. 2012;62(598):e314-21.

10. Overeem K, Lombarts MJMH, Arah OA, Klazinga NS, Grol RPTM, Wollersheim HC. Three methods of multi-source feedback compared: a plea for narrative comments and coworkers' perspectives. Med Teach. 2010;32(2):141-7.

11. Edwards A, Evans R, White P, Elwyn G. Experiencing patient-experience surveys: a qualitative study of the accounts of GPs. Br J Gen Pract. 2011;61(585):157-66.

12. Lockyer JM, Violato C, Fidler HM. What multisource feedback factors influence physician self-assessments? A five-year longitudinal study. Acad Medi. 2007;82(10 Suppl):S77-80.

13. Ferguson J, Wakeling J, Bowie P. Factors influencing the effectiveness of multisource feedback in improving the professional practice of medical doctors: a systematic review. BMC Med Educ. 2014;14:76.

14. Kluger AN, DeNisi A. The effects of feedback interventions on performance: a historical review, a meta-analysis, and a preliminary feedback intervention theory. Psychol Bull. 1996;119(2):254

15. Smither JW, London M, Reilly RR. Does performance improve following multisource feedback? A theoretical model, meta-analysis, and review of empirical findings. Pers Psychol. 2005;58(1):33-66.

16. Geissler KH, Friedberg MW, SteelFisher GK, Schneider EC. Motivators and barriers to using patient experience reports for performance improvement. Med Care Res Rev. 2013;70(6):621-35.

17. Miller A, Archer J. Impact of workplace based assessment on doctors' education and performance: A systematic review. BMJ. 2010;341(7775):1-6.

18. Pearson K: Taking revalidation forward: Improving the process of relicensing for doctors. Sir Keith Pearson's review of medical revalidation. In. http://www.gmc-uk.org/Taking_revalidation_forward Improving_the_process_of_relicensing_for_doctors.pdf_68683704.pdf: General Medical Council; 2017.

19. Moher D, Liberati A, Tetzlaff J, Altman DG. Preferred reporting items for systematic reviews and meta-analyses: the PRISMA statement. Ann Inter Med. 2009;151(4):264-9.

20. Khan KS, Ter Riet G, Glanville J, Sowden AJ, Kleijnen J: Undertaking systematic reviews of research on effectiveness: CRD's guidance for carrying out or commissioning reviews: NHS Centre for Reviews and Dissemination; 2001.

21. Booth A. Clear and present questions: formulating questions for evidence based practice. Library Hi Tech. 2006;24(3):355-68.

22. Sampson M, McGowan J, Cogo E, Grimshaw J, Moher D, Lefebvre C. An evidence-based practice guideline for the peer review of electronic search strategies. J Clin Epidemiol. 2009;62(9):944-52.

23. Rayyan - a web and mobile app for systematic reviews [https:// rayyan.qcri.org/]

24. Barr H, Freeth D, Hammick M, Koppel I, Reeves S: Evaluations of Interprofessional Education: A United Kingdom Review of Health and Social Care. London: CAPE; 2000.

25. CASP Qualitative Checklist [http://www.casp-uk.net/casp-tools-checklists]

26. Quality Assessment Tool for Observational Cohort and Cross-Sectional Studies [https://www.nhlbi.nih.gov/health-pro/guidelines/in-develop/ cardiovascular-risk-reduction/tools/cohort]

27. Quality Assessment of Systematic Reviews and Meta-Analyses [https://www nhlbi.nih.gov/health-pro/guidelines/in-develop/cardiovascular-risk-reduction/ tools/sr_ma]

28. Dixon-Woods M, Cavers D, Agarwal S, Annandale E, Arthur A, Harvey J, Hsu R, Katbamna S, Olsen R, Smith L, et al. Conducting a critical interpretive synthesis of the literature on access to healthcare by vulnerable groups. BMC Med Res Methodol. 2006;6(1):1-13.

29. Thomas J, Harden A. Methods for the thematic synthesis of qualitative research in systematic reviews. BMC Med Res Methodol. 2008;8(1):1-10.

30. Higgins JP, Green S: Cochrane handbook for systematic reviews of interventions, vol. 5: Wiley Online Library; 2008.

31. Dixon-Woods M, Bonas S, Booth A, Jones DR, Miller T, Sutton AJ, Shaw RL, Smith JA, Young B. How can systematic reviews incorporate qualitative research? A critical perspective. Qual Res. 2006;6:27-44.

32. Fereday J, Muir-Cochrane E. Demonstrating rigor using thematic analysis: A hybrid approach of inductive and deductive coding and theme development. Int J Qual Methods. 2006:5(1):80-92.

33. Popay J, Roberts H, Sowden A, Petticrew M, Arai L, Rodgers M, Britten N, Roen K, Duffy S: Guidance on the conduct of narrative synthesis in systematic reviews. A product from the ESRC methods programme Version 2006, 1:b92.
34. Farley $\mathrm{H}$, Enguidanos ER, Coletti CM, Honigman L, Mazzeo A, Pinson TB, Reed K, Wiler JL. Patient satisfaction surveys and quality of care: an information paper. Ann Emerg Med. 2014;64(4):351-7.

35. Ward CE, Morella L, Ashburner JM, Atlas SJ. An interactive, all-payer, multidomain primary care performance dashboard. J Ambul Care Manage. 2014;37(4):339-48.

36. Sargeant J, Bruce D, Campbell CM. Practicing physicians' needs for assessment and feedback as part of professional development. J Contin Educ Health Prof. 2013;33(S1):S54-62.

37. Paxton ES, Hamilton BH, Boyd VR, Hall BL. Impact of isolated clinical performance feedback on clinical productivity of an academic surgical faculty. J Am Coll Surg. 2006;202(5):737-45.

38. Gillam SJ, Siriwardena AN, Steel N. Pay-for-performance in the United Kingdom: Impact of the quality and outcomes framework-A systematic review. Ann Fam Med. 2012;10(5):461-8.

39. Yen L, Gillespie J, Jeon YH, Kljakovic M, Brien JA, Jan S, Lehnbom E, PearceBrown C, Usherwood T. Health professionals, patients and chronic illness policy: A qualitative study. Heal Expect. 2011;14(1):10-20.

40. Vivekananda-Schmidt P, MacKillop L, Crossley J, Wade W. Do assessor comments on a multi-source feedback instrument provide learner-centred feedback? Med Educ. 2013;47(11):1080-8.

41. American College of Obstetrics and Gynaecology, Committee on Patient Safety and Qualtiy Improvement and Committee on Health Care for Under Served Women, Opinion No. 587: Effective patient-physician communication. Obstet Gynaecol. 2014;123(2 Pt 1):389-93.

42. Hageman MG, Ring DC, Gregory PJ, Rubash HE, Harmon L. Do 360-degree feedback survey results relate to patient satisfaction measures? Clin Orthop Relat Res. 2015;473(5):1590-7.

43. Hess BJ, Lynn LA, Conforti LN, Holmboe ES. Listening to older adults: elderly patients' experience of care in residency and practicing physician outpatient clinics. J Am Ger Soc. 2011:59(5):909-15.

44. Lockyer JM, Violato C, Fidler HM. What multisource feedback factors influence physician self-assessments? A five year longitudinal study. Acad Med. 2007;82(10):577-80

45. Wilkie V, Spurgeon P. Translation of the Medical Leadership Competency Framework into a multisource feedback form for doctors in training using a verbal protocol technique. Educ Prim Care. 2013;24(1):36-44.

46. Elliott MN, Kanouse DE, Burkhart Q, Abel GA, Lyratzopoulos G, Beckett MK, Schuster MA, Roland M. Sexual Minorities in England Have Poorer Health and Worse Health Care Experiences: A National Survey. J Gen Intern Med. 2015;30(1):9-16.

47. Hrisos S, Illing JC, Burford BC. Portfolio learning for foundation doctors: Early feedback on its use in the clinical workplace. Med Educ. 2008:42(2):214-23.

48. Fowler L, Saucier A, Coffin J. Consumer assessment of healthcare providers and systems survey: Implications for the primary care physician. Osteopathic Fam Physician. 2013;5(4):153-7.

49. Fryers $M$, Young $L$, Rowland $P$. Creating and sustaining a collaborative model of care. Healthc Manage Forum. 2012;25(1):20-5.

50. Brinkman WB, Geraghty SR, Lanphear BP, Khoury JC, del Rey JAG, DeWitt TG, Britto MT. Effect of multisource feedback on resident communication skills and professionalism: a randomized controlled trial. Arch Pediatr Adolesc Med. 2007:161(1):44-9.

51. Rodriguez HP, von Glahn T, Elliott MN, Rogers WH, Safran DG. The effect of performance-based financial incentives on improving patient care experiences: a statewide evaluation. J Gen Intern Med. 2009;24(12):1281-8.

52. Fustino NJ, Kochanski JJ. Improving Patient Satisfaction in a Midsize Pediatric Hematology-Oncology Outpatient Clinic. J Oncol Practice. 11(5):416-20.

53. Violato C, Lockyer JM, Fidler H. Changes in performance: a 5-year longitudinal study of participants in a multi-source feedback programme. Med Educ. 2008:42(10):1007-13.

54. Reinders ME, Ryan BL, Blankenstein AH, van der Horst HE, Stewart MA, van Marwijk HW. The effect of patient feedback on physicians' consultation skills: a systematic review. Acad Med. 2011;86(11):1426-36.

55. Overeem K, Lombarts KM, Cruijsberg JK, Grol RP, Arah OA, Wollersheim HC: Factors predicting doctors' performance change in response to multisource feedback. Doctor performance assessment. 2012:89.

56. Nielsen JF, Riiskjær E. From patient surveys to organizational change: Rational change processes and institutional forces. J Chang Manage. 2013; 13(2):179-205.

57. Overeem K, Wollersheim H, Driessen E, Lombarts K, Van De Ven G, Grol R, Arah O. Doctors' perceptions of why 360-degree feedback does (not) work: a qualitative study. Med Educ. 2009;43(9):874-82. 
58. Sargeant J, Mcnaughton E, Mercer S, Murphy D, Sullivan P, Bruce DA. Providing feedback: exploring a model (emotion, content, outcomes) for facilitating multisource feedback. Med Teach. 2011;33(9):744-9.

59. Sargeant JM, Mann KV, Van der Vleuten CP, Metsemakers JF. Reflection: a link between receiving and using assessment feedback. Adv Health Sci Educ. 2009;14(3):399-410.

60. Sargeant J, Mann K, Sinclair D, Van Der Vleuten C, Metsemakers J. Challenges in multisource feedback: Intended and unintended outcomes. Med Educ. 2007;41(6):583-91.

61. Wentlandt K, Bracaglia A, Drummond J, Handren L, McCann J, Clarke C, Degendorfer N $\mathrm{N}$ Chan CK. Evaluation of the physician quality improvement initiative: the expected and unexpected opportunities. BMC Med Educ. 2015;15:230

62. Sargeant J, Bruce D, Campbell CM. Practicing physicians' needs for assessment and feedback as part of professional development. J Contin Educ Health Prof. 2013;33(Suppl 1):S54-62.

63. Ferguson J, Wakeling J, Bowie P. Factors influencing the effectiveness of multisource feedback in improving the professional practice of medical doctors: a systematic review. BMC Med Educ. 2014;14(76)

64. Gallan AS, Girju M, Girju R. Perfect ratings with negative comments: Learning from contradictory patient survey responses. Patient Experience J. 2017:4(3):15-28.

65. Robert Wood Johnson Foundation: Tell me a story: how patient narrative can improve health care. In. 2018:1-8.

66. Tan A, Hudson A, Blake K. Adolescent narrative comments in assessing medical students. Clin Teach. 2018;15:245-251. https://doi.org/10.1111/tct. 12667.

67. Ginsburg S, Regehr G, Lingard L, Eva KW. Reading between the lines: faculty interpretations of narrative evaluation comments. Med Educ. 2015;49(3): 296-306.

68. Burn S, D'Cruz L. Clinical audit--process and outcome for improved clinical practice. Dent Update. 2012;39(10):710-4.

69. Ramani S, Könings KD, Ginsburg S, van der Vleuten CPM. Twelve tips to promote a feedback culture with a growth mind-set: Swinging the feedback pendulum from recipes to relationships. Med Teach. 2018:1-7.

70. Ginsburg S, Vleuten CP, Eva KW, Lingard L. Cracking the code: residents' interpretations of written assessment comments. Med Educ. 2017;51(4): 401-10.

71. Archer JC. State of the science in health professional education: effective feedback. Med Educ. 2010;44(1):101-8.

72. Bawa-Garba - From blame culture to just culture [http://blogs.bmj.com/bmj/ 2018/04/06/david-nicholl-bawa-garba-from-blame-culture-to-just-culture/]

73. Ladher N, Godlee F. Criminalising doctors. BMJ. 2018;360:k479.

74. Holton EF. The flawed four-level evaluation model. Hum Resour Dev Q. 1996; $7(1): 5-21$

75. Yardley S, Dornan T. Kirkpatrick's levels and education 'evidence'. Med Educ. 2012;46(1):97-106.

76. Pawson R, Greenhalgh T, Harvey G, Walshe K. Realist review-a new method of systematic review designed for complex policy interventions. J Health Serv Res Policy. 2005:10(1 suppl):21-34.

77. Brennan N, Bryce M, Pearson M, Wong G, Cooper C, Archer J. Towards an understanding of how appraisal of doctors produces its effects: a realist review. Med Educ. 2017;51(10):1002-13.

78. Wong G, Greenhalgh T, Pawson R. Internet-based medical education: a realist review of what works for whom and in what circumstances. BMC Med Educ. 2010;10(12)

Ready to submit your research? Choose BMC and benefit from:

- fast, convenient online submission

- thorough peer review by experienced researchers in your field

- rapid publication on acceptance

- support for research data, including large and complex data types

- gold Open Access which fosters wider collaboration and increased citations

- maximum visibility for your research: over $100 \mathrm{M}$ website views per year

At $\mathrm{BMC}$, research is always in progress.

Learn more biomedcentral.com/submissions 